Estimating Parametric, Model Form, and Solution Contributions Using Integral Validation Uncertainty Quantification

R. W. Logan, C. K. Nitta, S. K. Chidester

March 17, 2006 
This document was prepared as an account of work sponsored by an agency of the United States Government. Neither the United States Government nor the University of California nor any of their employees, makes any warranty, express or implied, or assumes any legal liability or responsibility for the accuracy, completeness, or usefulness of any information, apparatus, product, or process disclosed, or represents that its use would not infringe privately owned rights. Reference herein to any specific commercial product, process, or service by trade name, trademark, manufacturer, or otherwise, does not necessarily constitute or imply its endorsement, recommendation, or favoring by the United States Government or the University of California. The views and opinions of authors expressed herein do not necessarily state or reflect those of the United States Government or the University of California, and shall not be used for advertising or product endorsement purposes.

This work was performed under the auspices of the U.S. Department of Energy by University of California, Lawrence Livermore National Laboratory under Contract W-7405-Eng-48. 


\title{
Estimating Parametric, Model Form, and Solution Contributions using Integral Validation Uncertainty Quantification
}

\author{
Roger W. Logan, Cynthia K. Nitta, Steven K. Chidester \\ University of California, Lawrence Livermore National Laboratory \\ email: RWLOGAN@LLNL.GOV, NITTA1@LLNL.GOV, \\ CHIDESTER1@LLNL.GOV
}

\begin{abstract}
$\underline{\text { Abstract }}$
One of the final steps in building a numerical model of a physical, mechanical, thermal, or chemical process, is to assess its accuracy as well as its sensitivity to input parameters and modeling technique. In this work, we demonstrate one simple process to take a top-down or integral view of the model, one which can implicitly reflect any couplings between parameters, to assess the importance of each aspect of modeling technique. We illustrate with an example of a comparison of a finite element model with data for violent reaction of explosives in accident scenarios. We show the relative importance of each of the main parametric inputs, and the contributions of model form and grid convergence. These can be directly related to the importance factors for the system being analyzed as a whole, and help determine which factors need more attention in future analyses and tests.
\end{abstract}

\section{Nomenclature}

\author{
DFSS Design For Six Sigma \\ FEA Finite Element Analysis \\ FEM Finite Element Method \\ PDF Probability Distribution Function \\ RSM Response Surface Model \\ S Simulation Output (Quantity of Interest) \\ S' Simluation Output sensitivity to a data input d \\ $\mathrm{u}_{\mathrm{d}} \quad$ Standard Uncertainty in data inputs $\mathrm{d}$ \\ $\mathrm{u}_{\mathrm{D}} \quad$ Standard Uncertainty in data output $\mathrm{D}$ (determined experimentally) \\ $\mathrm{u}_{\mathrm{IVAL}} \quad$ Standard Uncertainty from regression of model output $\mathrm{S}$ vs. data output $\mathrm{D}$ \\ $\mathrm{u}_{\mathrm{SDP}} \quad$ Standard Uncertainty in model output $\mathrm{S}$ due to Data Parametric uncertainty $\mathrm{u}_{\mathrm{d}}$ \\ $\mathrm{u}_{\mathrm{SMA}} \quad$ Standard Uncertainty in model output $\mathrm{S}$ due to Modeling Assumptions (Model Form) \\ $\mathrm{u}_{\mathrm{SN}} \quad$ Standard Uncertainty in model output $\mathrm{S}$ due to Numerical errors (solution verification) \\ $\mathrm{V} \& \mathrm{~V} \quad$ Verification \& Validation
}




\section{Introduction}

Model validation is the process of determining whether a model of a physical process is adequate from the perspective of the real world use of the model. This is true whether the model is a simple curve fit or a nonlinear finite element solution. Quantitative model validation typically involves uncertainty quantification, in order to develop quantitative measures of confidence intervals and prediction intervals over the range of validation of the model (comparison against test data), and outside the range of data (predictive capability estimates ${ }^{1}$ ). Two forms of validation are commonly used. These may be called Hierarchical and Integral validation. Hierarchical validation (also called bottom-up ${ }^{2}$ ) is described as a process where the model is built in steps, with uncertainty quantification at each level. Integral validation (also called top-down) is a process where a model of the top-level quantity of interest is built, and the model assessed against top level data. The hierarchical method provides more information about the way that subsystems and parametrics affect the final quantity of interest. Furthermore, hierarchical models are often less ad-hoc, so it is possible to achieve a highly physics-based model instead of a top level curve fit. The downside of the hierarchical method is time, cost, and possibly even drift, as bias errors in the hierarchical path may stack up to form a bias in the top level solution. Because of this, it is of value to use both hierarchical and integral model validation and uncertainty quantification. We find that integral models, while they may contain more free parameters, can be quickly compared to available referent data, and top level confidence bounds can be constructed if we account properly for the existence of free (fitting) parameters in the model ${ }^{3,4,5,6}$. The integral model is useful as a screening tool or a pilot to guide future work on more detailed, physically based hierarchical models. The integral model also provides a bias check; ideally, the mean and confidence bounds on the hierarchical and integral models should align very closely. If not, one or the other (or both) contain bias errors or uncertainties not properly quantified.

Even while using these "integral models" as screening and planning assessment tools, we desire to gain a feeling for the components of uncertainty contributed by each major factor in the model. For example, a finite element model will have uncertainties due to:

- [A] Plan simulations: Assess existing output data uncertainty, codes, code errors

- [B] Solution Verification (spatial, temporal, iterative convergence)

- [C] Parametric Variability (experimental scatter in the input quantities)

- [C] Parametric Uncertainty (lack of information - not enough data)

- [D] Model Form or Physics Uncertainty (lack of knowledge about the physics nature)

We have denoted each of these aspects with the letters A, B, C, or D, corresponding to an "ABCD" process for Model Verification \& Validation (V\&V) described in previous works ${ }^{17}$. The letters "ABCD" can represent both the process used for model $\mathrm{V} \& \mathrm{~V}$, but also reflect specific types of uncertainty terms which may be quantified using the same "ABCD" breakout. We can make a direct analogy between our interpretation of the "ABCD" process for assuring accurate FEA simulations, and one proposed process by Coleman and $\mathrm{Stern}^{8}$ for validation with uncertainties at confidence. For clarity we follow the nomenclature as proposed ${ }^{8}$, but will use standard uncertainties $(u)$ instead of 
expanded uncertainties (U) in this work, resulting in the following analogs and nomenclature ${ }^{8}$ :
$\mathrm{A} \sim \mathrm{u}_{\mathrm{D}} \sim$ Data uncertainty, experimental and measurement uncertainty in available output quantity data; may include small sample coverage factors ${ }^{9}$ or other corrections
$\mathrm{B} \sim \mathrm{u}_{\mathrm{SN}} \sim$ Simulation Numerical uncertainty, determined from spatial, temporal, and iterative convergence studies ${ }^{10}$
$\mathrm{C} \sim \mathrm{u}_{\mathrm{SPD}} \sim$ Simulation uncertainty in the model output quantity due to parametric uncertainties $u_{d}$ in the model input quantities
$\mathrm{D} \sim \mathrm{u}_{\mathrm{SMA}} \sim$ Simulation uncertainty due to Modeling Assumptions, or model form or model physics uncertainty

In this work, we use uncertainty quantification at the integral level to establish the overall uncertainty as a standard deviation of fit at the mean of the top level referent data available. We denote this quantity as $\mathrm{u}_{\mathrm{IVAL}}$, or the standard uncertainty of fit between the top level model and data output quantity. We assume a bias correction at the top level and so only consider explicitly the uncertainty terms above. In this simple treatment we will also assume for simplicity that the "ABCD" uncertainty terms can be assessed independently of each other. In the problem we present in this work that assumption is legitimate; however, such is not generally the case.

With an example in the main text and a description of a Crystal Ball example in the Appendix, we will show that "Six Sigma" principles are not just for production process control; there is an easy procedure to get started in applying Design For Six Sigma (DFSS) in FEA as well ${ }^{11}$. Along the way we will obtain the relative importance of our parametric inputs, grid convergence, and model form.

\section{Example of the "DFSS" process for FEA}

The problem selected involves the assessment of high explosive safety in possible accident scenarios. The assessment involves use of the Steven impact test ${ }^{12}$ (Figure 1). A steel shape impacts a sandwich assembly containing high explosive. At or above a certain impact velocity, the HE will begin to react. This "threshold" velocity is predicted by simple regression response surface models (RSM), and also by complex finite element models (FEM). The result is a plot of the mean threshold velocity versus, for example, the temperature of the assembly during impact (Figure 2). Confidence intervals (shown), prediction intervals, the integral validation uncertainty term $\mathrm{u}_{\mathrm{IVAL}}$, and reliability of the model of the system ${ }^{11,13}$ can be assessed from the top level with this type of integral model and validation ${ }^{14}$. 


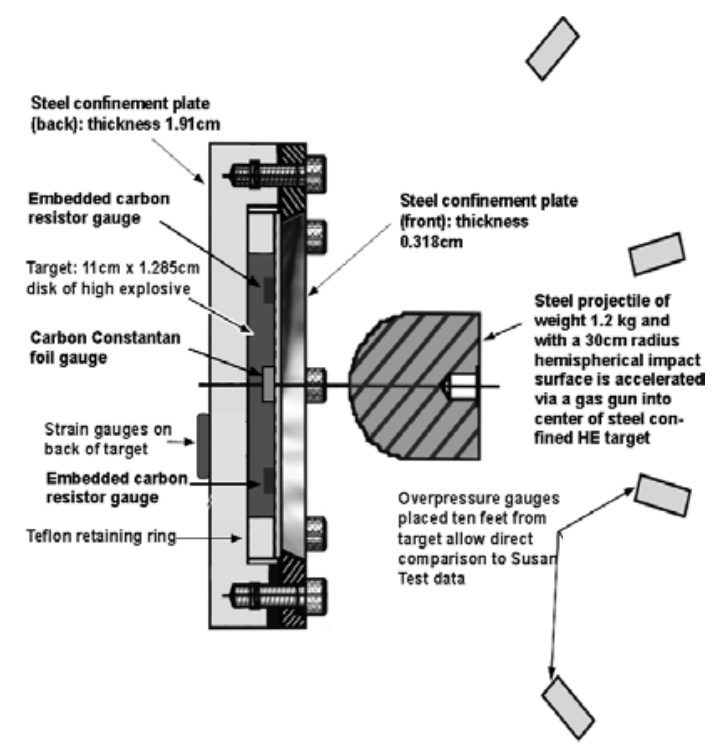

Figure 1. Steven Impact Test: 30mm (Steven, Head \#1, shown) and 6mm (Duff R/T, Head \#2, not shown) projectiles.

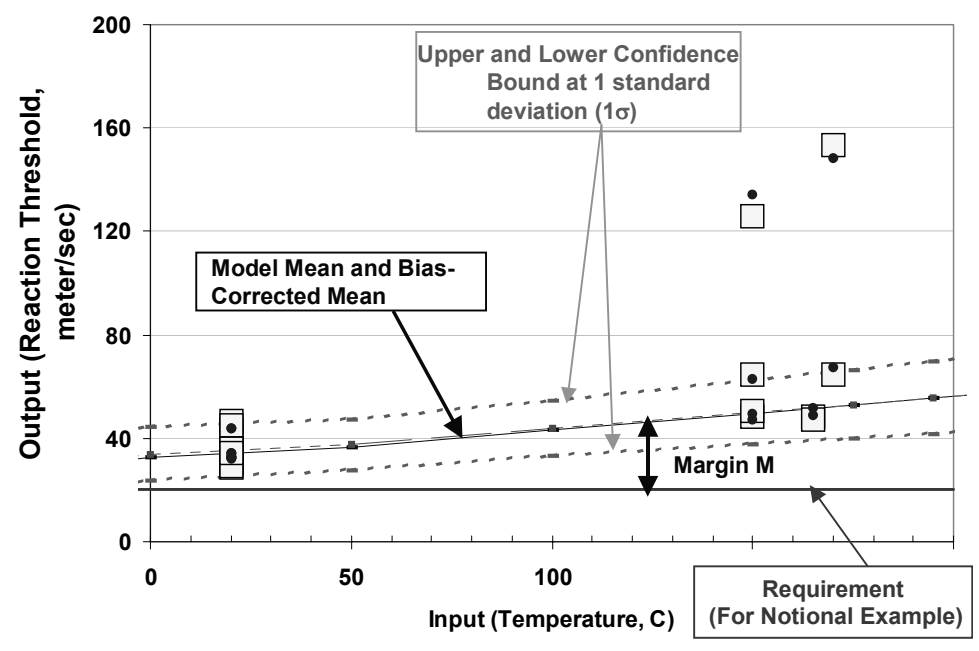

Figure 2. Integral Model Validation plot for PBX 9404 impact threshold, using 0D Regression model, showing mean and confidence intervals.

We then overlay probabilistic tools over our simulations to examine the separate contributions of solution verification $\left(\mathrm{u}_{\mathrm{SN}}\right)$ and parametric uncertainty $\left(\mathrm{u}_{\mathrm{SPD}}\right)$ estimates. Typically, during the development of either simple regression models or complex nonlinear finite element models, we find that the largest uncertainty contributor is the model physics or model form uncertainty. This point is illustrated in Figure 3. We assess the total standard deviation of fit, confidence interval, and prediction interval using the integral validation method, and plot this as the bar marked "all" as the total standard deviation of the fit versus the referent data, or $\mathrm{u}_{\mathrm{IVAL}}$. The probabilistic simulator is then 
used to sample known (or various assumed) probability distribution functions (PDFs) of the physical data used as input to the model. Each probabilistic simulation gives a slightly different reaction threshold velocity and hence a slightly different standard deviation of fit will result. This simulation parametric data uncertainty $u_{S P D}$ is already affecting the integral model result $\mathrm{u}_{\mathrm{IVAL}}$, so we must be careful not to double count. However, if the effect of the $u_{S P D}$ is small compared to the total uncertainty $u_{\text {IVAL }}$, we can assess it in this manner and then subtract out independent terms in quadrature. As shown in Figure 3, the contribution to threshold velocity of the experimental variability due to input uncertainties $u_{d}$ in test temperature (Texpt in Fig. 3), and in explosive energy content (HMX\% in Fig. 3), and in the output quantity of interest data $\left(\mathrm{u}_{\mathrm{D}}\right)$ experimental velocity measurement (Vexpt in Fig. 3), are all very small compared to the total standard deviation of fit ( $\left.\mathrm{u}_{\mathrm{IVAL}}\right)$. For a linear model, the input uncertainties $\mathrm{u}_{\mathrm{d}}$ could be simply multiplied by the respective sensitivities ( $S^{\prime}$ ) of simulation model output (S) on change $u$ ${ }_{\mathrm{d}}$ in model input, giving, when added in quadrature:

$$
\mathrm{u}_{\text {SPD }}^{2}=\left(\mathrm{S}_{\text {Texpt }}\right)^{2}\left(\mathrm{u}_{\mathrm{d}, \text { Texpt }}\right)^{2}+\left(\mathrm{S}_{\text {HMX\% }}\right)^{2}\left(\mathrm{u}_{\mathrm{d}, \mathrm{HMX} \%}\right)^{2}
$$

For models where inputs affect the output in a nonlinear way, we can link a probabilistic tool to the simulation (FEA or RSM) model, and obtain the components of $\mathrm{u}_{\mathrm{SPD}}$ without needing a constant sensitivitity $S^{\prime}$ to propagate uncertainty from input to output.

Assessments of the solution verification (grid convergence) uncertainty $\left(\mathrm{u}_{\mathrm{SN}}\right)$ can be performed using methods described by Roache ${ }^{10}$, or more complex methods described by Nitta and Logan ${ }^{15}$ and elsewhere. For our example problem, the terms in $\mathrm{u}_{\mathrm{SN}}$ are also small $^{16}$. This leaves the Model bar in Figure 3, representing $\mathrm{u}_{\mathrm{SMA}}$ as the single remaining and dominant contributor to the overall estimate of the standard deviation of the model of the system. Given the assumptions discussed above and in previous works, we can obtain one possible numerical assessment of model form uncertainty $\mathrm{u}_{\mathrm{SMA}}$ from our extension of the previous nomenclature ${ }^{8}$ as:

$$
u_{\text {IVAL }}^{2}=u_{D}^{2}+u_{S N}^{2}+u_{S P D}^{2}+u_{S M A}^{2}
$$

Since we have quantified each remaining term (with our assumptions as given above for this simple explanation), we can obtain an assessment of $u_{\mathrm{SMA}}$ directly from subtraction in quadrature:

$$
u_{\text {SMA }}^{2}=u_{\text {IVAL }}^{2}-u_{D}^{2}-u_{\text {SN }}^{2}-u_{\text {SPD }}^{2}
$$

Note that such an assessment is only possible because we have done the integral level validation to obtain $\mathrm{u}_{\mathrm{IVAL}}$; otherwise we would be unable to quantify either term or separate either term. This method represents one simple way of estimating model form uncertainty in the integral method. It is valuable to compare uncertainty analyses such as the one summarized in Figure 3, using alternate model forms, as a check that the estimate of model form uncertainty encompasses that actually observed when using alternate plausible models. 


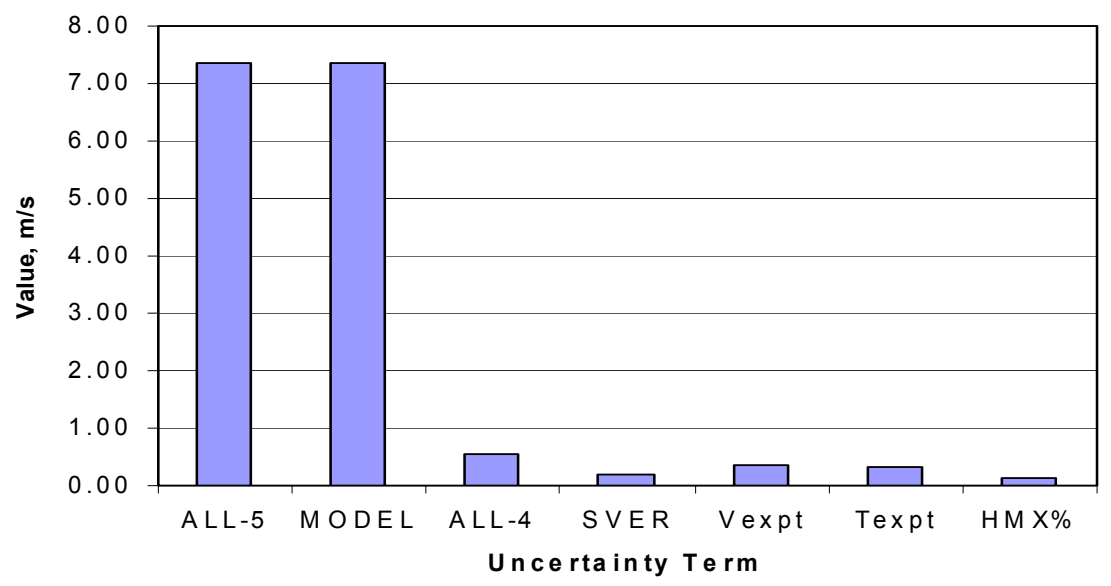

Figure 3. Uncertainty Quantification during integral validation: Model Physics uncertainty term dwarfs the other terms when they are extracted from the integral validation process.

The fact that the Model term dominates the experimental and solution verification terms tells us that lack of knowledge about the physical, chemical, and mechanical aspects of the physical impact problem are the largest limitation of the model. The compelling next phase is to improve the physics in the model and drive down the model uncertainty term until it is about the same magnitude as the other terms in Figure 3. It may or may not be possible to do this with the integral model. If it is not, then at least the integral model, combined with the ability to separate out the effects of variability using the probabilistic simulator overlay, has allowed us to show the relative magnitudes and that the model form or model physics uncertainty should be a top priority. This type of analysis may not enable the integral model to be improved; however, it makes a compelling and quantitative case to proceed with development of a detailed, albeit costly, hierarchical model, so that we may drive down the height of the model uncertainty bar in Figure 3.

\section{Conclusion}

We have divided the uncertainties during a non-deterministic FEA into four major terms, and shown an example method to quantify three types of terms in a top-down or integral analysis for the uncertainty present in the FEA model of the system. These three types of terms (solution verification, parametric uncertainty, and model form uncertainty) can be quantified to give a first but quantitative assessment of their relative importance. We offer an integral validation example (system level model versus data response) as a way to obtain an envelope estimate for model form uncertainty and recommend that this estimate be compared to that obtained using alternate plausible model forms. There will of course be many cases where many or most of the simplifying assumptions we follow here will be violated and cause inaccuracies in assessments of the model and system 
output. However, the simplicity of the treatment as presented elsewhere ${ }^{1-3,7-11}$ and followed here enables a clear comparison to more complex model analyses that may follow. Doing this type of analysis also enables each type of uncertainty at confidence (the "ABCD" or $\mathrm{u}_{\mathrm{D}}, \mathrm{u}_{\mathrm{SN}}$, $\mathrm{u}_{\mathrm{SPD}}, \mathrm{u}_{\mathrm{SMA}}$ terms) to be followed through to get a feel for their importance, notwithstanding coupling effects addressed in subsequent analyses. In addition, the breakout of the "ABCD" process and its analogy to the terms $\left(u_{D}, u_{S N}, u_{S P D}\right.$, $\mathrm{u}_{\mathrm{SMA}}$ ) reminds us to remember not to lose sight of the model form uncertainty term $\mathrm{u}_{\mathrm{SMA}}$; whether $\mathrm{u}_{\mathrm{SMA}}$ is quantified using integral validation as in this example or using hierarchical validation, the model form uncertainty $u_{\mathrm{SMA}}$ is always elusive, often dominant, and assessing it is paramount to the credibility of the use of the model in subsequent predictions.

\section{Acknowledgement}

This work was performed under the auspices of the U.S. Dept of Energy by Lawrence Livermore National Laboratory under contract W-7405-Eng-48. The authors wish to thank Lori Switzer, Kevin Vandersall, Daniel Greenwood, and Craig Tarver for their help in providing experimental results and theoretical assistance. We wish to acknowledge the work of Tony DePiero, David Sam, and Mark Garcia in solution verification, and the expert assistance of Henry Hsieh in the probabilistic aspects. 


\section{Appendix A: An Example with Crystal Ball}

Crystal Ball $^{1}{ }^{\circledR}$ is " a user-friendly, graphically oriented forecasting and risk analysis program that takes the uncertainty out of decision-making" (Quote from the user's manual). Briefly, Crystal Ball is a Sampling-Based (Monte Carlo or Latin Hypercube methods) probabilistic simulator that enables quantification, at a given Monte Carlo confidence level, of uncertainties propagated through Microsoft Excel $^{2}$ based models.

The Crystal Ball Example File, "Simulation with DoE.xls" can provide a simple example of the first use of the principles in this work. The example is described on the first 3 of the attached pages. On the next two pages, the actual spreadsheet is shown. For this weakly coupled problem, it is possible to examine the effects of the three parametric inputs, time, temperature, and pressure, do a simple root-sum-square combination, and get nearly exactly the same contribution as each is used in a Monte Carlo analysis separately or in combination. This gives us the estimate of parametric uncertainty. The model form uncertainty can be estimated via an integral least squares comparison of the model results versus experimental results. In this example we will neglect the solution verification uncertainty term.

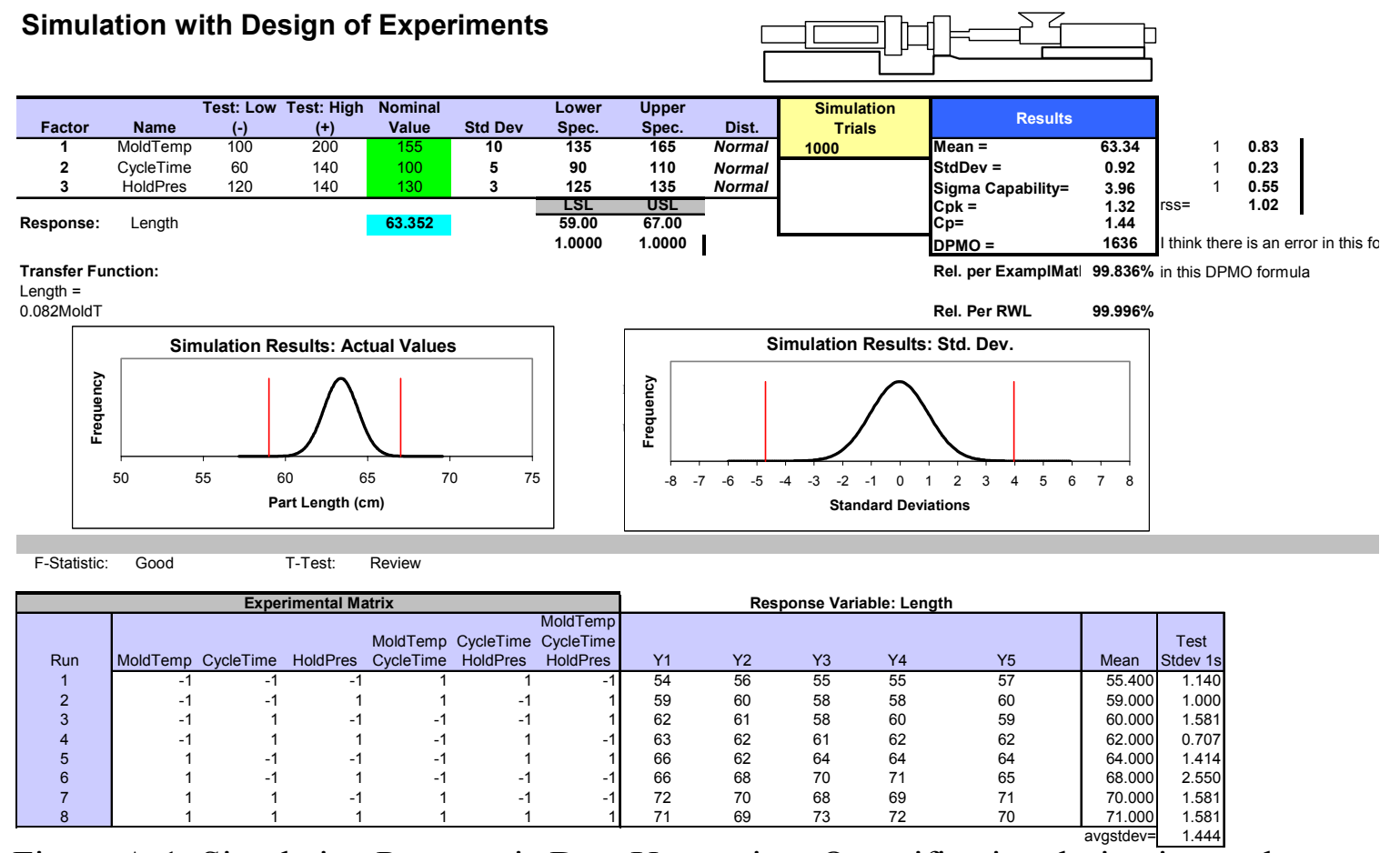

Figure A-1: Simulation Parametric Data Uncertainty Quantification during integral validation: This example, supplied with the Crystal Ball example set, has the same method of construction as the Steven Test example in the main text. This figure shows the Excel spreadsheet in the Crystal Ball example "Simulation with DoE.xls".

The resulting values are given in Figure A-2 for this Crystal Ball example. A similar procedure can be followed with direct links to a finite-element model instead of a simple

${ }^{1}$ Crystall Ball, C 1988-2004, by Decisioneering, Inc., 1515 Arapahoe St., Suite 1311, Denver, CO 80202

${ }^{2}$ Microsoft Excel, (C) Microsoft Corporation. 
analytical model as shown here. Such procedures are best followed using for example software such as NESSUS, DAKOTA, LS-OPT, or iSIGHT as noted in (Nitta and Logan, 2004) $)^{1}$.

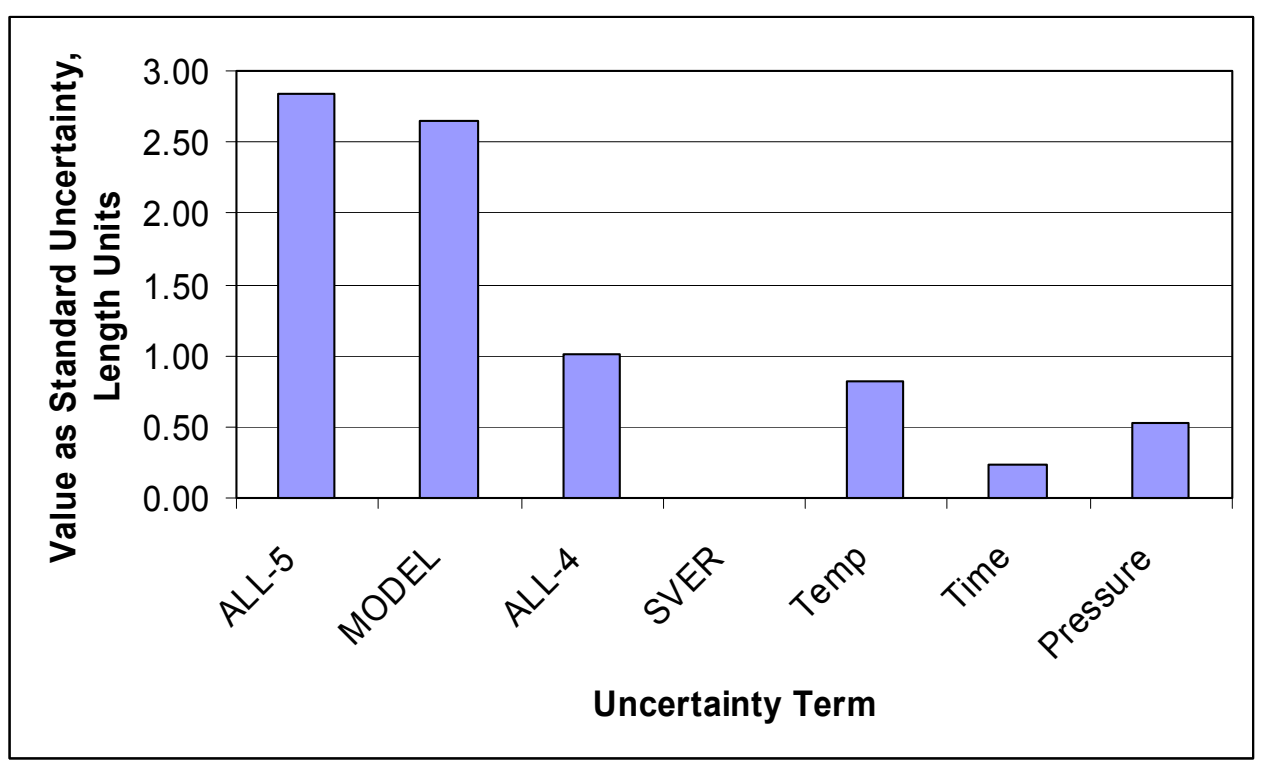

Figure A-2: Uncertainty Quantification during integral validation: This example, supplied with the Crystal Ball example set, has the same method of construction as the Steven Test example in the main text. The Crystal Ball Excel sheet shown in Figure A-1 can be used with a Response Surface Model of (or instead of) the FEA results, to quantify the components of the $u_{S P D}$ uncertainty term as standard uncertainties at 1-sigma confidence. 


\section{References}

${ }^{1}$ Nitta, C.K. and Logan, R.W., "Qualitative and Quantitative Linkages from V\&V to Adequacy, Certification, Risk, and Benefit / Cost Ratio", Lawrence Livermore National Laboratory LLNL UCRL-TR-205809, Sep 2004.

${ }^{2}$ Paez, T.L., Hinnerichs, T.D., Hasselman, T.K., and Wathugala, G.W., "Comparing Bottom-Up and Top-Down Approaches for Total Uncertainty Quantification", paper presented at $7^{\text {th }}$ U.S. National Congress for Computational Mechanics ( $7^{\text {th }}$ USNCCM), Albuquerque, NM, Jul 2003.

${ }^{3}$ Easterling, R.C., "Statistical Foundations for Model Validation: Two Papers", Sandia National Laboratories SAND2003-0287, Sandia National Laboratories, Feb 2003.

${ }^{4}$ Bevington, P.R. and Robinson, D.K., "Data Reduction and Error Analysis for the Physical Sciences", McGrawHill, 1969.

${ }^{5}$ Press, W.H., Teukolsky, S.A., Vetterling, W.T., and Flannery, B.P., "Numerical Recipes in C”, Cambridge University Press, 1992.

${ }^{6}$ Draper, N.R. and Smith, H., “Applied Regression Analysis”, Wiley \& Sons, 1981.

${ }^{7}$ Trucano, T.G., Pilch, M., and Oberkampf, W.L., "General Concepts for Experimental Validation of ASCI Code Applications", Sandia National Laboratories SAND-2002-0341, Mar. 2002.

${ }^{8}$ Coleman, H.W. and Stern, F., "Uncertainties in CFD Code Validation", ASME J. Fluids Eng., Vol. 119, pp. $795-$ 803, Dec 1997.

9 ASME, “Test Uncertainty", Performance Test Codes PTC 19.1, an American National Standard, ASME, New York, NY, Dec 1998

${ }^{10}$ Roache, P.J., "Quantification of Uncertainty in Computational Fluid Dynamics", Annual Review of Fluid Mechanics, Vol. 29, pp123-160, 1997.

${ }^{11}$ Logan, R.W., Nitta, C.K., and Chidester, S.K., "Design For Six Sigma with Critical-To-Quality Metrics for Research Investments", Lawrence Livermore National Laboratory LLNL UCRL-TR-214481, Aug. 2005.

${ }^{12}$ Switzer, L.L., Vandersall, K.S., Chidester, S.K., Greenwood, D.W., and Tarver, C.M., "Threshold Studies of Heated HMX-Based Energetic Material Targets Using the Steven Impact Test", $13^{\text {th }}$ APS Topical Conference on Shock Compression of Condensed Matter, Portland, OR, Jul 2003.

${ }^{13}$ Urbina, A., Paez, T.L., Gregory, D.L., and Resor, B.R., "Response Comparisons for Mechanical Joint Model Validation", Proc. $9^{\text {th }}$ ITEA Modeling \& Simulation Conference, Las Cruces, NM, Dec 2003.

${ }^{14}$ Nitta, C.K., Logan, R.W., Chidester, S.K., and Foltz, M.F., "Benefit / Cost Ratio in Systems Engineering: Integrated Models, Tests, Design, and Production", Lawrence Livermore National Laboratory LLNL UCRL-TR207610, Nov 2004.

${ }^{15}$ Nitta, C.K. and Logan, R.W., "Solution Verification Linked to Model Validation, Reliability, and Confidence", Lawrence Livermore National Laboratory LLNL UCRL-CONF-206544, Sep. 2004.

${ }^{16}$ DePiero, A.H., "Methodology for Predicting HE Ignition due to Low-Pressure, High-Deformation Impacts (U)", Lawrence Livermore National Laboratory LLNL CODTU-00-0149, proc. $5^{\text {th }}$ LS-DYNA Users Conference, Southfield, MI, Sep. 1998. 